\title{
Knowledge, Awareness and Associated factors of Telemedicine Services among Health Professionals Working at Amhara Region Referral hospitals, northwest Ethiopia, 2020.
}

Bayou Tilahun Assaye ( $\sim$ bayutilahun5@gmail.com )

Debre Markos University

Adamu Takele Jemere

University of Gondar

Araya Mesfin Nigatu

University of Gondar

\section{Research Article}

Keywords: Knowledge, Awareness, Telemedicine, Health Professionals, Amhara Region, Ethiopia

Posted Date: March 17th, 2021

DOI: https://doi.org/10.21203/rs.3.rs-334058/v2

License: (c) (i) This work is licensed under a Creative Commons Attribution 4.0 International License.

Read Full License 


\section{Abstract}

Background: -Telemedicine is the delivery of healthcare services at a distance. Despite it is an emerging and infancy technology in a developing country, utilizing the technology for delivering healthcare services is low in Ethiopia. To maximize and facilitate Telemedicine adoption it prominently requires information about the knowledge and awareness of telemedicine services among health professionals.

Methods: - An institution-based cross-sectional study design was conducted among 423 health professionals working at Amhara Region Referral Hospitals from February 12-March 20/2020. The data were collected using a self-administered structured questionnaire. Desk review was done to assure the data quality and then data was entered into EPI INFO version 7 and exported into SPSS version 20 for statistical analysis. Descriptive statistics, bivariable and multivariable logistic regression analyses were done. The odds ratio with a $95 \%$ confidence interval $(95 \% \mathrm{Cl})$ was used to identify associated factors.

Result: About $56 \%$ of health professionals had good knowledge and $57.4 \%$ had high awareness towards telemedicine services with a $411(97.2 \%)$ response rate. Information sharing culture [AOR=3.01, 95\% Cl: $1.89,4.80]$, having IT support staff [AOR=1.87, 95\% Cl: 1.06, 3.29], internet as information source $[\mathrm{AOR}=1.80,95 \% \mathrm{Cl}: 1.1,2.94]$, awareness [AOR=1.35,95\% Cl: $1.03,2.40]$, being male [AOR=1.73,95\% Cl:1.06, 2.81] were significantly associated with the knowledge of the respondents towards telemedicine services and telemedicine training [AOR=2.33,95\% Cl: $1.15,4.72]$ and computer accessibility in their hospitals [AOR=1.54, 95\% Cl: $1.01,2.35]$ were significantly associated with the awareness of the respondents towards telemedicine services.

Conclusion: More than half of the respondents had good knowledge and high awareness of telemedicine services. Information sources, having IT support staff, information sharing culture, gender and awareness were significant factors for the knowledge of telemedicine service, and telemedicine training and computer access were significant factors of awareness of health professionals towards telemedicine services. Therefore Appropriate and regular awareness creation training of telemedicine systems should be given to the health professional.

\section{Background}

Electronic health (eHealth) services are expanding rapidly and have the potential to improve the health of the community, enhance scientific understanding of health issues, and facilitating the communication between health care providers and patients (1-6). Telemedicine is an important tool for the amplification of healthcare delivery using smartphones, email, video conferencing, and especially in rural areas, use electronic information and communication technology to provide and support clinical care remotely (7). It ranges from a simple telephone conversation between providers up to a real-time videoconferencing involving doctors and patients (8-10).

Exchanging healthcare information and providing health care services across a geographic area and time has been considered as a potential solution to alleviate the current health care problem, revolutionize the 
health care system, and delivering to the rural and remote areas for disease control and prevention (11, 12). The diffusion and implementation of telemedicine services will ultimately depend on the knowledge and awareness of its application (13).

Accessing modern health care, getting specialty services is low and still limited due to different factors like poor infrastructures, individual ICT exposure's and information sharing cultures between health professionals or healthcare organizations, and there are further challenges for delivering health service due to rising communicable and non-communicable disease, the scarcity of medical specialists will not be overturned in a short time, inadequate transportation infrastructure makes it even more difficult to provide healthcare services in remote and rural areas where more than $80 \%$ of the population lives (1416). But integrating healthcare service delivery with information communication technology are challenges in Ethiopia's health system $(9,17-19)$.

The telemedicine system is an initiative and encouraging, by WHO in health system digitization and it is essential for Ethiopia as the country experiences a heavy burden of disease and a shortage of trained health professionals $(12,20-22)$.

The potential of telemedicine services in health care delivery was known, but particularly in developing countries, failed to sustain and integrate with the health care system. As the different studies indicated widely mentioned cause of failure is the level of knowledge and awareness of health professionals towards telemedicine services and understanding of health professionals, towards telemedicine is indeed critical to its sustainable development for health system digitization $(1,11,18,23-25)$.

To maximize the adoption of telemedicine services and sustainable development of health system digitization, user-oriented development of advanced systems integrating knowledge and awareness of telemedicine services among health professionals is necessary.

\section{Methods And Materials}

\section{Study Design, period and setting}

An institution-based cross-sectional study design was conducted at Amhara Region Referral Hospitals from February 12-March 20/2020. The State of Amhara consists of 13 administrative zones and the capital city is Bahir-Dar. It is the 2nd populous region in Ethiopia having about thirty Million people which accounts for $27 \%$ of the Ethiopian population. More than $85 \%$ of the Amhara population lives in rural areas. It is bordered by Sudan to the west and northwest, Benishangul-Gumuz to the west and southwest, Tigray to the north, Afar to the east, and Oromia to the south. There are six Referral Hospitals in the Amhara region and 4234 health professionals working on these Referral Hospitals.

All health professionals working at Amhara Region referral hospitals were included. Those Health professionals who were seriously ill and those who had less than six months of working experience in clinical practices were excluded from the study. 


\section{Sample size and sampling procedures}

\section{Sample size determination}

The sample size was determined by using single population proportion formula, $\mathrm{P}=50 \%$ of the health professionals to determine knowledge of telemedicine service and $52.6 \%$ of health professionals for had high awareness telemedicine services (11), $95 \% \% \mathrm{Cl}, 5 \%$ margin of error, and $10 \%$ non-response rate, the calculated sample size for knowledge $n_{1}=423$ and awareness $n_{2}=422$. So, the maximum sample 423 was taken as the final sample size.

\section{Sampling procedures}

Study participants were selected using a stratified sampling method followed by a proportional allocation from Amhara Region Referral Hospitals Northwest Ethiopia. First for each referral Hospitals and department, proportional allocations were done and then participants were selected using a simple random sampling method (Figure 1)

\section{Operational definition}

Good Knowledge: good knowledge of telemedicine service was defined as for those study participants who scored more than or equal to 9 (50\%) of knowledge (18 items yes or No) questions(26).

High awareness towards telemedicine services: was defined as for those study participants who scored more than or equal to 2.2 of 5 or $44 \%$ of awareness questions (five points Likert scale) that involves perceiving, feeling, opinion, and overview towards telemedicine services (27).

\section{Data Collection Procedures and Data Quality assurance Data collection procedures}

A self-administered structured questionnaire was used. The questionnaire was prepared in English. The questionnaire consists of socio-demographic characteristics, related to ICT exposures, organizationalrelated questions, 18 items either 'yes' or 'No ' was used to measure the health professionals' knowledge towards telemedicine services. Ten items with a five-point Likert scale ranging from 1-5 i.e. ' 1 ' for very little,' 2 ' for little,' 3 ' for some ' 4 ' for enough, and ' 5 ' for very enough was used. One can score a minimum of ' 10 ' and a maximum of ' 50 ' related to measure the awareness of the study participant and telemedicine services-related questions.

\section{Data quality assurance}

Before the actual data collection, a pre-test was done on $5 \%$ of the study participants outsides of the actual study area, the Cronbach alpha value of (0.81) knowledge and (0.76) awareness and modifications were done based on the pre-test.

The data collectors and the supervisor were given one-day training before participating in the data collection process to create awareness about respondents, the purpose of the study, their rights, and 
confidentiality issues. Sufficient time was be given to respondents for reading and filling materials carefully. There was Continuous supervision up to the end of data collection. After collecting the data, the supervisor and the investigator checked its consistency and completeness.

\section{Data Processing and Analysis}

Data were entered using Epi-info version 7 and analyzed using Statistical Package for Social Science (SPSS) version 20. Descriptive analyses were performed to describe the study population about relevant variables. The adjusted odds ratio with a $95 \%$ confidence interval was used to measure the association of dependent and independent variables. Bivariable logistic regression was conducted and those factors with $p$-value $<0.2$ were fitted into multivariable logistic regression analysis and variables with P.value $<0.05$ were considered as statistically significant in the multivariable logistic regression model. The Hosmer-Lemeshow test was used to test the model fitness and also multicollinearity was checked between independent variables.

\section{Ethical consideration}

Ethical clearance was obtained from the University of Gondar Institute of Public Health ethical review board and a support letter from Amhara Region Referral Hospitals. Written consent was obtained from each participant.

\section{Results}

\section{Socio-demographic characteristics}

A total of 423 study participants were selected from six Amhara Region Referral Hospitals for the assessment of knowledge and awareness of telemedicine services among health professionals working in the Amhara region. Four hundred eleven ( $97.2 \%$ response rate) of them were written consented and responded to complete all the questionnaires. Among the study participants, 258(62.8\%) of the respondents were male, the mean age of the participants was 29.65+_4.5 SD years and the majority of the respondents were within the age group of 20-29 years. In terms of educational status, most of the respondents were bachelor degree 268 (65.2\%). Regarding the professional category's the respondents 78 (19.0\%) medical doctors and $137(33.3 \%)$ were nurses. The mean working experience was 5+_3.3 SD years and more than half $242(58.9 \%)$ of the respondents were within the range of $1-5$ years (Table 1$)$.

\section{Exposure of ICT on the knowledge and awareness of telemedicine services}

About 249 (60.6\%) of the respondent just had an introductory level of ICT exposure, 334 (81.3\%) use computer or laptop or smartphone for their work, and from those $306(91.6 \%)$ used for internet access. Almost half $198(48.2 \%)$ of the participants sometimes search information for health care digitations, but $148(36.0 \%)$ of the respondent had not used internet application for telemedicine service at all (Table 2). 
According to this study majority of the respondents, 262 (64.0\%) had no sufficient computers for their work, $260(63.3 \%)$ of the respondent had internet access within their hospitals and $152(58.2 \%)$ of them has Wi-Fi types of internet access. Similarly 219 (53.3\%) of the respondent of health professionals had an information-sharing culture with other health care providers or patients and only $47(11.4 \%)$ of the study participant was attend training on telemedicine systems (Figure 2).

\section{Knowledge of health professionals by the types of telemedicine services}

Among study participants on the types of telemedicine services $151(36.7 \%)$ of the respondent knew of communication via telephone, 117 (28.47\%) of Store and forward, 104 (25.3\%) Remote monitoring, and 63 (15.33\%) Online/live (Figure 3).

\section{Clinical application area, common public health area, benefits, and Barriers to the knowledge and awareness of telemedicine services}

From the total study participants, the majority 317 (77.1\%) had awareness of telemedicine clinical application for Radiology and $212(51.6 \%)$ had awareness on the common public health application area of telemedicine services was for school-based health centers.

In this study, the knowledge of telemedicine services among health professional at referral hospitals 286 $(69.6 \%)$ of them knew the benefits of telemedicine system to improve the quality of healthcare system and $229(55.7 \%)$ of the respondent to send the patient for better treatment to another hospital (Table 3).

\section{Factors associated with the knowledge of telemedicine services among health professionals.}

The variables including gender, Having IT support staff, Information sharing culture, Information source and the awareness of telemedicine services were positively associated with knowledge of telemedicine services among health professionals working at Amhara Region Referral Hospitals.

According to this study, gender was significantly associated with the knowledge of telemedicine services among health professionals. Being male were 1.73 times more likely to have a knowledge of telemedicine services than female (AOR=1.73, 95\% Cl: [1.06-2.81]). Similarly, health professionals who have IT support staff in their hospitals were 1.87 times more likely knowledgeable on telemedicine services than those health professionals who had no IT support staff (AOR=1.87, 95\%Cl: [1.06-3.29]).

Information sharing culture was another factor for the knowledge of telemedicine services among health professionals. Health professionals with having information-sharing culture were 3 times more likely to have good knowledge of telemedicine services than those with no information-sharing culture (AOR=3.01, 95\% Cl: [1.89-4.80]).

Information source was one of the factors that positively associated with knowledge of telemedicine services. Health professionals with using the internet as an information source was were 1.80 times more likely than the counterpart ( $\mathrm{AOR}=1.80,95 \% \mathrm{Cl}$ : $11.10-2.94])$. Additionally, Health professional's awareness 
of telemedicine services was strongly associated with the knowledge of telemedicine services. Health professionals who had awareness of telemedicine was 1.35 times more likely knowledgeable than those who had (AOR=1.35, 95\% Cl [1.03-2.40]) (Table 4).

\section{Factors associated with the awareness of telemedicine services among health professionals}

In this study, training on the telemedicine system was significantly associated with the awareness of telemedicine services. Those who attended training on the telemedicine system had 2.33 times more likely to have the awareness of telemedicine services than those who did not attend the training (AOR= $2.33,95 \% \mathrm{Cl}:[1.15-4.72])$.

Similarly, the accessibility of computers in their hospitals was another factor affecting the awareness of telemedicine services. Health professionals who had computer access in their hospitals were 1.42 times more likely awarded than those who had no computer access in their hospitals ( $\mathrm{AOR}=1.42,95 \% \mathrm{Cl}$ : $[1.01$ 2.35]) (Table 5).

\section{Discussion}

To maximize the adoption of telemedicine services, the user-oriented development of advanced systems integrating knowledge and awareness of telemedicine services among health professionals is necessary $(5,28-32)$.

The study was conducted at six Amhara Region referral hospitals and assessed the knowledge, awareness, and associated factors of telemedicine services among health professionals. From this study, $56 \%$ (95\% Cl: [50.6, 59.9]) of health professionals had a good knowledge of telemedicine services. This study is in line with the study done in Saudi Arabia 53.9\% (7); however, this study result is higher than the study conducted in Nigeria 34.1\% (20) and India (41\%) (30). The possible explanation for this might be due to the sample size difference (the sample size of the study in Nigeria was 110 and in India 124), the study period. Also, currently, the Ethiopian ministry of health has been given great attention for health care digitization to be one of the main priority areas.

The study indicated that gender was significantly associated with the knowledge level; males were more likely knowledgeable than females. This study is in line with a descriptive study conducted in Bangladesh (30). The possible explanation might be: males have higher exposure to use internet access (33) and to new technology due to their literacy level compared to females (34).

Health professionals who have IT, support staff, in their hospitals were 1.9 times more likely to be knowledgeable on telemedicine services than those who didn't (AOR=1.87, 95\% Cl: [1.06-3.29]). This finding is supported by the study conducted in Kenya (35) and Ethiopia (36). The possible reasons might be due to the existing technology which can be realized by motivation and support by the end-users and the proper understanding of telemedicine technology is a way to scale-up the healthcare system. 
The finding of the study indicated that $38.7 \%$ of health professionals had a health information-sharing culture and were 3 times more likely knowledgeable towards telemedicine services than those who had no health information-sharing culture. This result was comparable with the study done in Austria (31). This could be due to telemedicine needs the willingness of the two ends (sender and receiver). This study also revealed that the majority $(88.4 \%)$ of respondents said that they have never attended any formal training on telemedicine. This finding was supported by the study done in Bangladesh ( $82.5 \%$ of them never attended any formal training on telemedicine) (30). Whereas study done in India found that none of the respondents had any formal training on telemedicine (32).

Information source was another statistically significant factor for knowledge of telemedicine services. Using the internet as an information source was about 1.8 times more likely to be knowledgeable than other sources. This study shows that the majority of the respondent reported that the internet $(33.1 \%)$ and different medical literature (21.6\%) were the main sources of information about telemedicine, followed by $15 \%$ of the respondent from colleagues and $9.9 \%$ from TV/Radio. This finding is contrasted with the study conducted in Bangladesh. In Bangladesh, the source for information for telemedicine was colleagues (38\%) and the internet (17.5\%) (30). The possible explanation might be due to the accessibility of the internet, healthcare digitization, and availability of different information technology and software applications.

Awareness was also the associated factor with knowledge of telemedicine services. In this study, health professionals who had awareness of telemedicine services were 1.35 times more likely to be knowledgeable than those who had no awareness. This finding indicated that more than half of the respondents (57.4\%) were aware of the benefits of telemedicine in terms of improving quality of care, access and convenience, cost reduction, and ensuring safety. Clinical application areas such as dermatology, radiology, and cardiology were priority areas identified by study participants.

The majority (65.2\%) of them knew Store-and-forward telemedicine, and communication via telephone about the types of telemedicine service deliveries, related to telemedicine application. Radiology, cardiology, and dermatology were indicated by $13.5 \%, 11.8 \%$, and $11.4 \%$ of the respondents respectively. Similarly, the option was given to respondents to choose among benefits of telemedicine systems; $69.6 \%$ of them identified that the system improves the quality of care, $58.2 \%$ indicated that it improves the healthcare access and $54.3 \%$ of the respondents indicated the system benefits in ensuring safety and security of patient information.

About 229 (55.7\%) of respondents replied that they would prefer sending the patient for better treatment to another hospital when encountered difficulties in their clinical work, $175(42.6 \%)$ of the respondents prefer to give patients appointments to come back when better physicians will be available and 162 $(39.4 \%)$ prefer to refer medical literature and/ or similar previous cases. This result is higher than the study conducted in Addis Ababa (11). Almost half (49.5\%) of the respondents were aware of the benefit of Telemedicine application, Radiology is indicated by $12.7 \%$ dermatology by $8.3 \%$ and Cardiology is 
chosen by $8.3 \%$ of the respondents. Similarly, $24.4 \%$ of the respondents recognized that the system improves the quality of care and $5.1 \%$ indicated that it only improve access and convenience.

Regarding the awareness level of health professionals, $57.4 \%$ (95\% Cl: $[53.0,63.5])$ of study participants had a high awareness of telemedicine services. This study is in line with the study conducted in Nigeria $58.5 \%$ (20), India 63\% (32) but lower than the study conducted in Uganda (70\%) (37). The probable reason could be due to infrastructure difference, shortage of health professionals trained with health care digitization as compared to the study area. Moreover, the basic computer ICT skills of health professionals might also contribute to the difference.

Health professionals who have sufficient access to computers in their institution relatively have had high telemedicine service awareness. This Study is in line with a study conducted in Addis Ababa (38). Health professionals who trained in telemedicine systems had high awareness towards telemedicine services, this study is lower than the study conducted In India (32).

The finding of this study revealed that more than $80 \%$ of the study participant had the awareness of telemedicine clinical application area which is higher than the study done in Tikur Anbesa hospital (52.6\%) (11).

\section{Conclusion}

More than half of the respondents had good knowledge and high awareness of telemedicine services. The Information source, having IT support staff, information sharing culture, gender, and awareness were the most significant factors for the knowledge of telemedicine service. Telemedicine training and computer access were factors affecting the awareness of health professionals towards telemedicine services. Therefore Appropriate and regular awareness creation training of telemedicine systems should be given to the health professional.

\section{Abbreviations}

BSC: Bachelor of Science; E-Health: Electronic Health; Epi-info: Epidemiological Information; ETB: Ethiopian Birr; FMOH: Federal Ministry of Health; GP: General Practitioners; HI: Health Informatics; HIT: Health Information Technician; HP: Health professionals; MPH: Master of Public Health; ICT: Information communication technology; IT: Information technology; SPSS: Statistical Package for Social Science; TGSRH: Tibebe Ghion Specialized Referral Hospitals; UGSRH: University of Gondar Specialized Referral hospital; WHO: World Health Organization ;

\section{Declarations}

\section{Ethics approval and consent to participate}


Ethical clearance was obtained from the University of Gondar institute of public health's ethical review committee. Official letters of support were obtained from the University of Gondar institute of public health and Communicate with the different official administrators of the referral hospitals. Written consent was obtained from each study participant after telling the objective of the study. The data collection procedure was anonymous.

\section{Consent for publication}

Not applicable

\section{Availability of data and materials}

All major data have been presented in the manuscript.

\section{Conflicts of Interest}

The authors declare that they have no conflicts of interest.

\section{Funding}

The University of Gondar funded the study. The funding body has no role in the design of the study and collection, analysis, interpretation of the data, in writing the manuscript and publication as well.

\section{Authors' Contributions}

BT substantially contributed to the conception and design, analysis, and interpretation of the study. AT and $A M$ were involved in the analysis, interpretation and all authors participated in the write-up of the article. All authors approved the manuscript for publication

\section{Acknowledgment}

We would like to acknowledge the University of Gondar Institute of Public Health Department of Health Informatics and Amhara Region Referral Hospitals for their permission, data collectors, and study participants for their support in the process of data collection.

\section{Author's information}

${ }^{1}$ Department of Health informatics, College of Health Sciences, Debre Markos University, P.0.Box 269, Email: bayutilahun5@gmail.com.

${ }^{2}$ Department of Health informatics, Institute of Public Health, College of Medicine and Health Sciences, University of Gondar, P.O.Box 196, Email: adamutakele@gmail.com, Gondar, Ethiopia. ${ }^{2}$ Department of Health informatics, Institute of Public Health, College of Medicine and Health Sciences, University of Gondar, P.O.Box 196, Email: hitdt2005@gmail.com, Gondar, Ethiopia. 


\section{References}

1. Parvin R, Shahjahan MJJotlSfT, eHealth. Knowledge, attitude, and practice on e-health among doctors working at selected private hospitals in Dhaka, Bangladesh. 2016;4:e15 (1-1).

2. Bali S. Barriers to Development of Telemedicine in Developing Countries. Telemedicine: IntechOpen; 2018.

3. Boring M, Waghray S, Lavanya R, Babu DBG, Badam RK, Harsha N, et al. Knowledge and awareness of teledentistry among dental professionals-A cross-sectional study. 2015;9(8): ZC41.

4. Eye MJAER. The digital divide in the utilization of Information and Communication Technology (ICT) in counselor education in Nigerian Universities. 2014;11(2):91-102.

5. García-Lizana F, Giorgo FJJot, telecare. The future of e-health, including telemedicine and telecare, in the European Union: from stakeholders' views to evidence-based decisions. 2012;18(6):365-

6. Ibrahim MIM, Phing C, Palaian SJJCDR. Evaluation of knowledge and perception of Malaysian health professionals about telemedicine. 2010;4:2052-7.

7. Albarran Al, Mohammed R, Almarshoud N, Almujalli L, Aljaeed R, Altuwaijiri S, et al. Assessment of physician's knowledge, perception and willingness of telemedicine in Riyadh region, Saudi Arabia. 2019.

8. El-Mahalli AA, El-Khafif SH, Al-Qahtani MFJPihimA, American Health Information Management Association. Successes and challenges in the implementation and application of telemedicine in the eastern province of Saudi Arabia. 2012;9(Fall).

9. Tambo E, Madjou G, Mbous Y, Olalubi OA, Yah C, Adedeji AA, et al. Digital health implications in health systems in Africa. 2016;3(1):91-3.

10. Wernhart A, Gahbauer S, Haluza DJPo. eHealth and telemedicine: Practices and beliefs among healthcare professionals and medical students at a medical university. 2019;14(2):e0213067.

11. Bisrat A. KNOWLEDGE AND PERCEPTION OF HEALTH CARE PROVIDERS TOWARDS TELEMEDICINE APPLICATIONS \& BENEFITS:: A Survey from Tikur Anbessa \& Nekemete Hospitals: Addis Ababa University; 2010.

12. FMoH E. Health Sector Transformation Plan. HSTP 2015/16-2019/20. August 2015.

13. Lindholm K, Ekeland AG, Jensen LK, Rasmussen J, Pedersen CD, Bowes A, et al. A model for assessment of telemedicine applications: mast. 2012;28(1):44-51. 
14. Tuot DS, Boulware LEJAickd. Telehealth applications to enhance CKD knowledge and awareness among patients and providers. 2017;24(1):39-45.

15. Magdalena M, Bujnowska-Fedak UGB, Grata-Borkowska UJShT, telehealth. Use of telemedicinebased care for the aging and elderly: promises and pitfalls. 2015;3:91-105.

16. Mars MJYomi. Health capacity development through telemedicine in Africa. 2010;19(01):87-93.

17. Price M, Williamson D, McCandless R, Mueller M, Gregoski M, Brunner-Jackson B, et al. Hispanic migrant farm workers' attitudes toward mobile phone-based telehealth for management of chronic health conditions. 2013;15(4):e76.

18. Sadoughi F, Hemmat M, Valinejadi A, Mohammadi A, Majdabadi HAJIJoCS, Security N. Assessment of Health Information Technology Knowledge, Attitude, and Practice among Healthcare Activists in Tehran Hospitals. 2017;17(1):155.

19. Tomar D, Menon I, Tyagi U, Singh A, Goyal J, Singh RJJDS. Assessment of awareness about teledentistry among dentists in a private dental college of Muradnagar, Ghaziabad. 2018;6:8-12.

20. Abodunrin O, Akande TJIJoHR. Knowledge and perception of e-health and telemedicine among health professionals in LAUTECH teaching hospital, Osogbo, Nigeria. 2009;2(1).

21. Crowley D, Delargy IJHRJ. A national model of remote care for assessing and providing opioid agonist treatment during the COVID-19 pandemic: a report. 2020;17(1):1-5.

22. Jennett $P, Y e o M$, Pauls $M$, Graham J. Organizational readiness for telemedicine: implications for success and failure. Journal of telemedicine and telecare. 2003;9 Suppl 2:S27-30.

23. Asemahagn MAJBhsr. Knowledge and experience sharing practices among health professionals in hospitals under the Addis Ababa health bureau, Ethiopia. 2014;14(1):431.

24. Lonergan PE, Washington III SL, Branagan L, Gleason N, Pruthi RS, Carroll PR, et al. Rapid utilization of telehealth in a comprehensive cancer center as a response to COVID-19: Cross-sectional analysis. 2020;22(7):e19322.

25. Mburu S, Kamau OJJoHliA. Framework for Development and Implementation of Digital Health Policies to Accelerate the Attainment of Sustainable Development Goals: Case of Kenya eHealth Policy (2016-2030). 2018;5(2).

26. Biruk K, Abetu EJJohe. Knowledge and Attitude of Health Professionals toward Telemedicine in Resource-Limited Settings: A Cross-Sectional Study in North West Ethiopia. 2018;2018.

27. Sheikhtaheri A, Sarbaz M, Kimiafar K, Ghayour M, Rahmani SJECiHAISAPoM. Awareness, Attitude, and Readiness of in Mashhad, Iran. 2016;228:142. 
28. Barr PJ, Brady SC, Hughes CM, McElnay JCJJoeicp. Public knowledge and perceptions of connected health. 2014;20(3):246-54.

29. Bhowmik D, Duraivel S, Singh RK, Kumar KSJTPI. The telemedicine-an innovating healthcare system in India. 2013;2(4, Part A):1.

30. ESTA DIRJG. Knowledge and Attitude of The Physicians Toward Telemedicine. 2017;5(12):85.

31. Wernhart A, Gahbauer S, Haluza DJPo. eHealth and telemedicine: Practices and beliefs among healthcare professionals and medical students at a medical university. 2019;14(2).

32. ZayapragaSSaraZan Z, Kumar SJJoc, JCDR dr. Awareness, knowledge, attitude, and skills of telemedicine among health professional faculty working in teaching hospitals. 2016;10(3): JC01.

33. Andualem M, Kebede G, Kumie A. Information needs and seeking behavior among health professionals working at public hospital and health centers in Bahir Dar, Ethiopia. BMC Health Serv Res. 2013;13:534-.

34. Shiferaw KB, Mehari EA. Internet use and eHealth literacy among health-care professionals in a resource-limited setting: a cross-sectional survey. Adv Med Educ Pract. 2019;10:563-70.

35. Mburu S, Oboko R. A model for predicting utilization of mHealth interventions in low-resource settings: the case of maternal and newborn care in Kenya. BMC Med Inform Decis Mak. 2018;18(1):67-.

36. Mengesha GH, Garfield MJ. A contextualized IT adoption and use model for telemedicine in Ethiopia. Information Technology for Development. 2019;25(2):184-203.

37. Kibera VM, Scott RE, Mars M. Assessing core, e-learning, clinical and technology readiness to integrate telemedicine at public health facilities in Uganda: a health facility-based survey. BMC Health Serv Res. 2019;19(1):266.

38. Biruk S, Yilma T, Andualem M, Tilahun BJBmi, making d. Health Professionals' readiness to implement electronic medical record system at three hospitals in Ethiopia: a cross-sectional study. 2014;14(1):115.

\section{Tables}

Due to technical limitations, Tables 1-5 are only available as a download in the Supplemental Files section

\section{Figures}




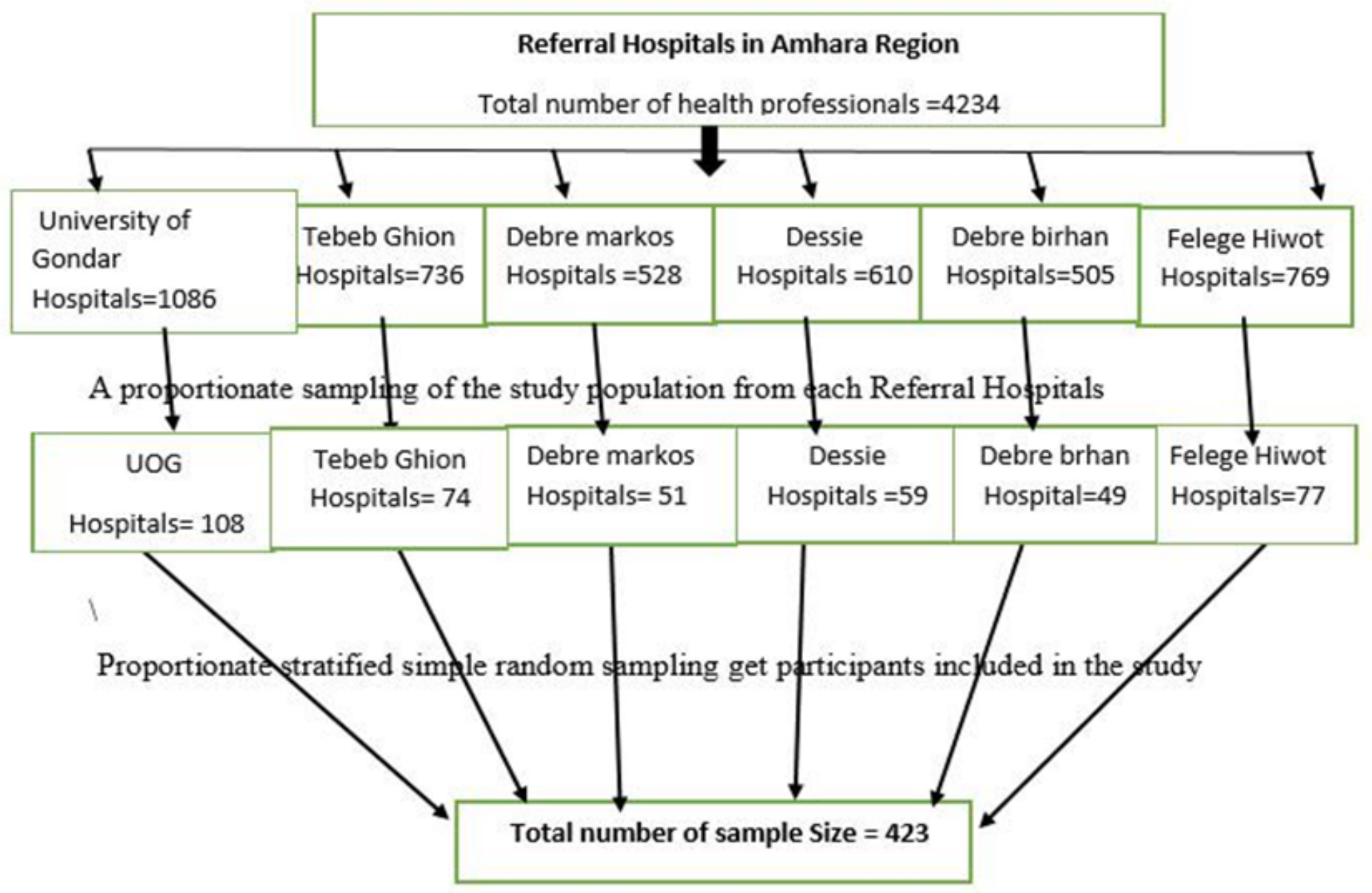

Figure 1

Sampling procedure for Health Professionals at Amhara Region Referral Hospitals, 2020 400

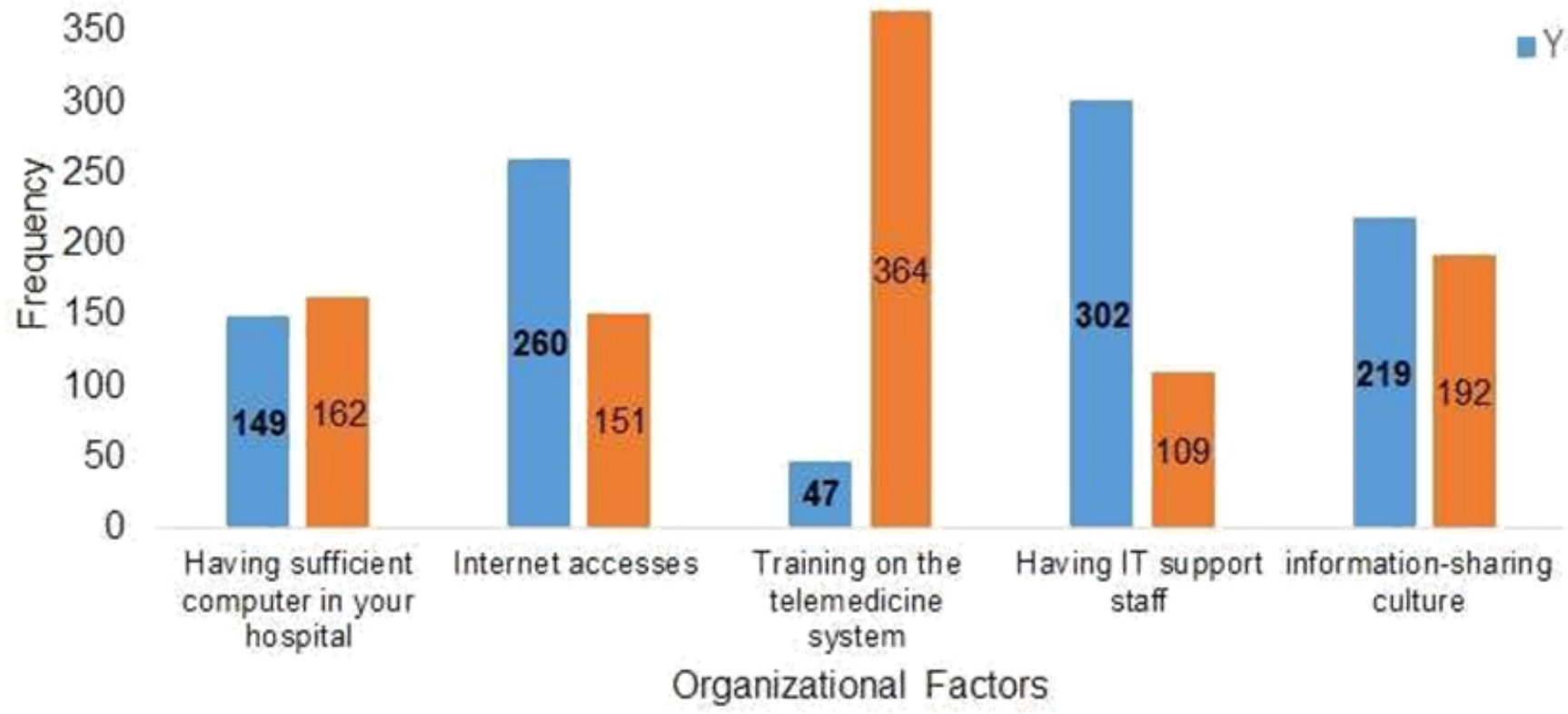

Figure 2 
Organizational factors on the knowledge and awareness of telemedicine services among health professionals at Amhara Region Referral Hospitals northwest Ethiopia, 2020.

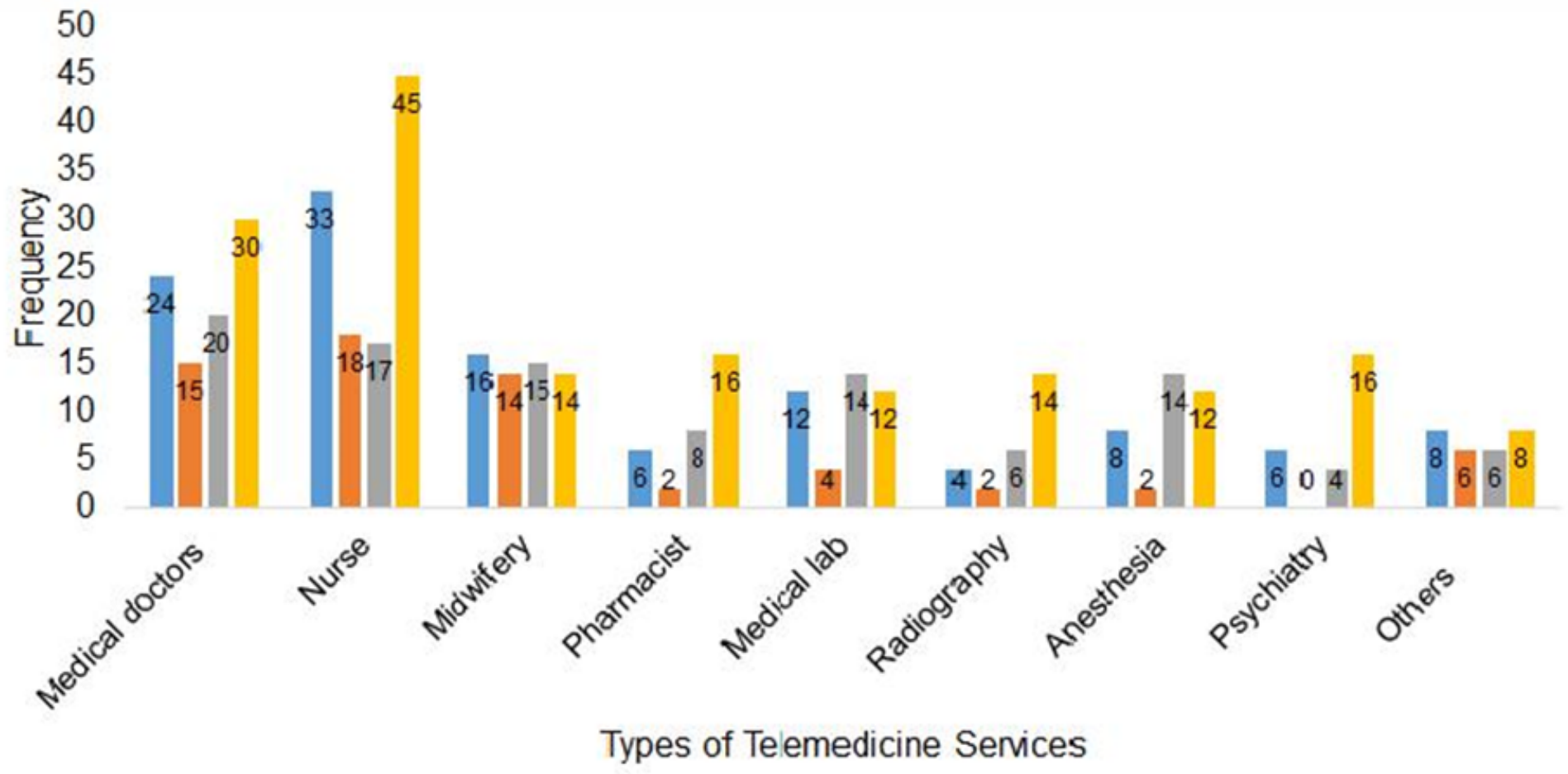

a Store and forward video-conferencing a Remote nonitoring a Communication via telephone

\section{Figure 3}

Knowledge of health professionals by the types of telemedicine services among the health professional at Amhara Region referral Hospitals, northwest 2020.

\section{Supplementary Files}

This is a list of supplementary files associated with this preprint. Click to download.

- Tables.docx 